\title{
L'écriture romanesque : une projection spatiotemporelle chez Cung Giu Nguyen
}

\author{
Pham Van Quang \\ Université nationale du Vietnam à HMC-Ville
}

Qu'est-ce que je fais? je saute, je marche, je nage, je foule, je piétine; mon vocabulaire devient inadéquat, aucun mot ne correspond plus à la complexe et fugace réalité, aucun mot ne garde sa signification privilégiée qui lui donne l'unicité, l'efficience ou la beauté à moins que le mot ne dénote le confus, le vague, le vide, le non-sens, l'anti-mot, le pré-mot, le non-mot, l'a-mot, les maux.

Cung Giu Nguyen, Le Boujoum

Que peut-on écrire de l'écrivain francophone vietnamien Cung Giu Nguyen au moment où l'espace de la réception de son 
œuvre semble toujours être mis en question et que cette fortune créatrice en tant qu'empreinte du temps risque d'être oubliée sur cette ancienne péninsule indochinoise?

Telle est la question qui soulève notre attention à l'égard de la trajectoire littéraire de Cung Giu Nguyen, une des grandes figures de la littérature vietnamienne d'expression française. En effet, force est de se souvenir des tribulations éprouvées par l'écrivain en tant que sujet social et sujet de l'écriture qui connut très tôt un état d'exil intérieur. Aussi l'interrogation précitée pourrait-elle nous amener à une double constatation : soit l'image de l'écrivain disparaîtrait sur la scène de la littérature nationale, tel son personnage d'Amdo dans $L e$ Boujoum, avec un sentiment de doute de son existence et du langage qui n'est plus pour lui pertinent dans la communication; soit cette interrogation inaugure un nouvel horizon permettant d'ébaucher un portrait manifeste de cet écrivain francophone.

Nous sommes, pour notre part, persuadé que les lecteurs prétendant défendre et promouvoir des valeurs patrimoniales des œuvres créatrices souhaiteraient que le soleil luise toujours à l'horizon lointain de l'ancienne Indochine et que l'image de Cung Giu Nguyen s'inscrive de plus en plus profondément dans l'esprit du public de son pays et du monde. D'autant plus que ce romancier prouve sans cesse sa foi en l'art par le moyen duquel il invente sa propre voie : il s'est efforcé de donner à la vie une signification, surtout au moment où sa communauté a été confrontée à des problèmes politiques et sociaux implacables. 


\section{Malédiction collective et individuelle : de la réalité à l'écriture}

À la lecture de l'œuvre romanesque de Cung Giu Nguyen, ce qui frappe particulièrement, c'est que l'auteur y évoque habilement une spatiotemporalité dotée de caractéristiques redoutables, précaires et opaques. Mais sur le plan fictif, comment peut-on expérimenter une spatiotemporalité aussi opaque et fugace? Sans doute fallait-il vivre assez longtemps pour pouvoir ressentir et saisir cette fugacité de l'espace existentiel et du temps solitairement éprouvant. Plus que tout autre, Cung Giu Nguyen se révèle capable de pénétrer ce domaine au point qu'il a passé tout un siècle à méditer sur son existence et à observer le cours de la société en se sentant entouré d'une atmosphère, paradoxalement, familière et inaccessible. Cette situation antagoniste vécue est constatée dans une lettre de Raoul Serène, un ami de l'écrivain, à propos du roman Le Boujoum : «Probablement, je vois justement que l'auteur se promenait dans son âme et qu'il vivait dans une circonstance quasiment inconnue tout en cherchant à se transcender» (Cung Giu Nguyen, 1994, p. VII ; je traduis).

Cette «circonstance inconnue» implique ce que nous nommons la spatiotemporalité dans l'œuvre romanesque de Cung Giu Nguyen ; c'est aussi ce que nous tentons d'examiner par les notions de temps et d'espace à travers l'acte d'écriture romanesque. En effet, l'auteur même, longtemps attaché à l'étude philosophique, comprend mieux que personne la notion de temps ainsi que son rapport avec l'être humain. Si le temps est une projection du conscient, son existence accorde à l'écrivain une possibilité de se reconnaître plus intimement, que le temps se caractérise consciemment ou inconsciemment. Dans 
l'œuvre romanesque de Cung Giu Nguyen, le temps est souvent abordé comme une perturbation et comme une implacabilité qui met le sort au défi. Certes, la structure romanesque exige des éléments fantastiques, mais en réalité, le texte romanesque transmet une projection psychologique, une auto-réflexion ou un refoulement. Déjà, Freud formule clairement cette idée quand il prend en considération la dimension psychanalytique de l'écrivain en tant que sujet de l'écriture :

Le roman psychologique doit en tout point sa particularité à la tendance de l'écrivain moderne à cliver son moi, par autoobservation, en mois partiels et à personnifier en conséquence les courants conflictuels de sa vie psychique en plusieurs héros. (Assoun, 1996, p. 42)

Le clivage du moi et la personnification des courants conflictuels apparaissent donc sous forme de projections du conscient. Cung Giu Nguyen produit certes une écriture fantastique, mais c'est dans cette écriture que l'auteur témoigne d'une cristallisation, d'une concentration des réalités auxquelles il s'est heurté, pour les transformer finalement en inconscient. La naissance de son œuvre romanesque marque ainsi une procédure d'expulsion et un itinéraire transcendant qui nous permettent de la lire comme un accès à un monde fantastique où s'exprime d'une manière pertinente le refoulement de la vie mal-entendue qui aurait pour corollaire la "rêverie éveillée »; on peut également y apercevoir un long rêve d'angoisse.

Par un effet de conceptualisation, aux personnages de ses romans sont assignées des réalités sensibles de façon à évoquer de façon légitime la dimension du temps vécu par l'écrivain et le glissement des expériences réelles vers l'écriture fantastique. L'image de Mô, personnage principal du Fils de la baleine, en est 
un exemple emblématique : il est l'expression des temps de changements, de confrontations et de contradictions. Aussi le protagoniste se constitue-t-il lui-même comme objet de changements générés par des conflits.

Sur le plan de la connotation, le nom propre Mô renvoie à rien en vietnamien : ce nom signifie la « nullité ». Mais peut-être ce vide de sens du terme et cette nullité assignée au personnage répondent-ils au choix et à la stratégie de l'auteur pour mettre en évidence la fragilité et l'absurdité de l'être humain, d'une part, et pour faire allusion à une projection illusoire, à une confusion des temps dont l'apport est le sujet précaire et solitaire, d'autre part. Si le sujet devient le lieu où passe la transition de la tradition à la modernité, il représente aussi le temps du désarroi. Mô incarne ainsi le sujet vivant dans ce chaos des conceptions de l'individuel et du collectif, de la tradition et de la modernité, du «moi » et du «nous», de la raison et du sentiment, du reconnu et du refusé :

Souvent le doute le saisissait. Les hommes lui montraient la même hostilité ou la même indifférence. Tous, du plus petit au plus vieux, n'avaient jamais voulu croire à ce qu'il disait. Détenaient-ils la vérité ? Tout ce qu'il trouvait dans sa tête n'était-il que vives illusions? Le passé auquel il pensait appartenir ne s'attachait peut-être à aucune vérité. Cent personnages pouvaient habiter en lui. Lequel correspondait à son moi véritable ? (Cung Giu Nguyen, 1978, p. 131).

L'écriture romanesque participe, pour ainsi dire, du langage d'une cure de l'état d'opacité intérieure. Les courants conflictuels ont lieu dans la personne du protagoniste. C'est ainsi que le sujet de l'écriture se divise dans ce personnage comme une sorte de projection du moi. En d'autres termes, par le biais de Mô, Cung Giu Nguyen initie le lecteur à la découverte 
de son propre moi clivé. L'écrivain transmet donc dans son écriture une expression de son univers intérieur, une projection de son moi en correspondance avec l'expression psychologique du personnage. Cependant, cette projection du moi clivé est déchiffrée au moyen des représentations psychiques du personnage. Aussi le temps des confusions détermine-t-il en grande partie le caractère de l'individu dans l'espace social qui façonne nécessairement l'identité du personnage social, comme cela s'exprime concrètement par le personnage de Mô. Se révèle par conséquent une dialectique entre réel et fantasme, entre fiction et cadre spatiotemporel, ce qui représente le processus de transformation du moi : du sujet social, par le sujet de l'écriture, au personnage romanesque. Cette analyse du moi peut se mettre en application aussi bien sur le plan du signifié que sur celui du signifiant, c'est-à-dire que nous pouvons prendre le texte romanesque comme le matériau référant au fantasme de l'auteur. En effet, les motifs récurrents dans l'œuvre de Cung Giu Nguyen permettent de supposer des situations dramatiques concernant l'individu et la communauté sociale à laquelle appartient le romancier. Ce point de vue psychocritique vise finalement la mise en évidence du mythe personnel de l'écrivain en corrélation avec sa vie réelle. Ainsi donc, chez l'écrivain, la vie et l'écriture trouvent leur point commun dans « la rêverie éveillée ».

Toujours sur le plan de la description du temps en rapport avec le récit de vie dans un espace donné, l'œuvre romanesque de Cung Giu Nguyen médite sans cesse sur les caprices du temps qu'affronte la condition humaine. Il en va ainsi, dans Le Domaine maudit, du personnage de Loan, qui vit dans la douleur causée par les confrontations idéologiques de l'époque. Le temps romanesque s'est forgé de manière logique à 
partir des caractéristiques ou des catégories de la temporalité : temps social, temps cosmique et temps personnel (une sorte de projection du conscient). Le temps social, comme nous l'avons vu pour le cas du personnage de Mô, annonce le temps intérieur, univers psychique consciemment vécu. Il s'agit du temps capable de régir et de remotiver les activités du personnage. Le Domaine maudit décrit en fait le temps des conflits sociaux, non sur le plan de la tradition et de la modernité, mais sur celui des conceptions idéo-politiques choisies individuellement. Dans cette perspective, la scénographie sociale, enchevêtrée, est décrite dans le roman par des schémas narratifs d'opposition, par le système syntactico-sémantique d'antagonisme. Les confrontations qui s'exercent au sein de la famille montrent la crise des valeurs traditionnelles et institutionnelles. Monsieur Quang, le père de famille, représente ces valeurs ancestrales, quelque peu aristocratiques, et prétend les éterniser, alors que Minh, son fils, renonce à ces valeurs établies et dénonce même le mode de vie aristocratique pour s'engager dans son propre chemin, qui mène à la révolution communiste. Loan, quant à elle, la fille aînée, regarde désespérément la vie s'écouler, car dans sa position de femme, il est impossible pour elle de poursuivre l'idéal inachevé de son père, la construction d'un arbre généalogique. Aussi s'agit-il d'un présage de solitude, d'angoisse : « Le train poursuivait sa course monotone et, plus d'une fois, Loan revit le film de ses dernières années. » (Cung Giu Nguyen, 1961, p.10) Par ce regard rétrospectif, l'auteur évoque chez le personnage une époque dramatique qui s'allonge jusqu'à l'horizon lointain ainsi que l'expérience d'un traumatisme psychique. L'écrivain précise encore le devenir du destin : 
La solitude l'enfermait avec son angoisse. Aucune issue ne lui offrait le moindre secours. Le monde se détournait d'elle, le monde l'abandonnait à son sort. Le monde vaquait à ses affaires, le monde allait en prison, le monde allait à la messe, le monde conduisait les automobiles, le monde faisait des comptes. Elle seule devrait surmonter sa peur, avaler l'amertume de sa vie. Le soleil se couchait, le soleil se levait. Les nuages passaient dans le ciel. La pluie tombait pour ne plus tomber. Mais, pour elle, de quoi demain serait-il fait? (ibid.)

«De quoi demain serait-il fait?»: cette question suggère le prélude lointain de la précarité du destin et circonscrit en même temps un regard prospectif avec l'impression d'un itinéraire inquiétant. Ce passage montre également qu'il y a corrélation entre le temps social et le temps cosmique, ce qui met en évidence, d'ailleurs, le pauvre sujet qui plonge dans son univers plein de figures humaines, mais vide de relations et de communications entre elles. Avec ce type d'existence humaine, Cung Giu Nguyen met en intrigue les éléments cosmiques et sociaux dans leur progression comme la trame faisant ressortir le processus psychique d'un moi solitaire.

\section{Au paroxysme de la solitude ou une quête d'existence profonde}

S'agissant du temps social et du temps cosmique vécus par l'écrivain, Cung Giu Nguyen évoque un temps chaotique dans $L e$ Boujoum, son troisième roman. En effet, dès l'incipit, le lecteur a l'impression d'une certaine hésitation chez le sujet de l'écriture, qui retarde l'entrée en scène du personnage. Plus précisément, l'auteur met le personnage dans un embarras dans sa définition par rapport à l'espace et au temps, comme s'il était marqué par une descente en abyme de l'univers, et dans cette initiation, lors 
du retour du conscient, il s'interroge sur le passage du temps : il y a des marques temporelles précises dans l'écriture mais elles ne progressent pas de manière chronologique selon le temps cosmique objectivement observé ; le temps scientifique ne peut occulter le temps de la Genèse :

Apesanteur, banalité dans un monde où les trouvailles newtoniennes deviennent absurdes et ridicules. Mais chez cet illuminé qui ouvrit une ère d'éclairs de génie la pomme échappée de la branche se refuserait à choir. Pomme d'Adam qui ne chuta que pour être jetée dans une autre sphère où tous les corps doivent nécessairement tomber. (Cung Giu Nguyen, 2002, p. 11)

La hantise du temps revient comme un état psychique chez le sujet en quête du passé et pris par la nostalgie : «cette sustentation pour une durée dont les termes sont certains et qu'on voudrait inexistants, ou tout au moins lointains » (ibid.). Cette obsession du temps se transforme dans l'inconscient pour engendrer l'illusion du temps immuable ou du temps en suspens : "Quelqu'un ne remonte-t-il pas la pendule pour que le temps ensorcelé ne se dévide plus ?» (ibid.). Cette question révèle une allusion à un chaos de l'univers qui ne fonctionne plus selon notre perception, car « la nuit n'est pas différente du jour ", ou bien, on se demande si, dans cette confusion, «le temps s'en est allé ailleurs pour que les gens qui auraient soif de plus d'être à chaque perte de souffle le réaniment » (ibid.).

Par le jeu sur le topos de l'inconnu à propos du protagoniste, Cung Giu Nguyen accède à la fiction dans la plus grande confusion, ouvrant sur l'évocation d'une série d'interrogations sur le temps, la dimension existentielle et l'aspiration au temps chez le sujet. En effet, si l'homme devient le centre où passe le temps, cette expérience du temps 
imprègne pleinement le sujet de l'écriture. C'est aussi par là qu'on voit le lien entre le temps de l'Histoire et l'espace de l'existence. L'Histoire et l'existence menacent le destin humain et, le plus souvent, elles entraînent une amertume et un anéantissement :

La terre bien travaillée ou retournée des entrailles devient traîtresse; on s'imaginait heurter des blocs durs, piétiner des institutions, des civilisations, on découvre de moins en moins une assise stable où poser tranquillement un derrière humain. La déception est rapide, mais pas autant que l'effondrement. (Cung Giu Nguyen, 2002, p. 12.)

Le temps de l'Histoire concourt à construire l'inconscient social et le fantasme individuel. À travers le temps de l'Histoire et le cadre scénographique, le roman ouvre un espace immense où le sujet de l'écriture se procure de la liberté pour déterminer ses rapports avec les expériences vécues et avec son fantasme. Il semble donc évident que le contexte historique est reformulé de manière condensée dès les premières pages du roman, ce qui amène à penser que le processus romanesque est une "recherche du temps perdu», comme le remarque Marthe Robert (1972, p. 68). Ce processus met en évidence le temps historique lié au temps personnel, qui se réduit dans le texte à une forme d'art de la représentation d'un passé obsédant. Comme un espace intime, le roman permet à l'écrivain d'essayer de faire revivre le temps en conceptualisant le personnage d'Amdo comme celui qui est sensible aux échos du passé perdu. À travers son personnage, Cung Giu Nguyen expose son temps individuel en référence au temps social, mais dans un univers imaginaire. Certes, Le Boujoum n'est pas un roman réaliste, mais on y trouve des relations complexes avec le monde sensible en ce que le discours romanesque remet en 
cause le discours idéologique de l'époque, ce qui permet de se rendre compte de la participation du romancier à l'inconscient social lorsqu'il s'interroge sur le vide et l'insignifiant du point de vue du temps et de l'étant: "Ai-je vécu en mangeant des alphabets Morse?» (Cung Giu Nguyen, 2002, p.13) Il s'agit d'une supposition de la naissance du personnage et de l'existence du sujet créateur. Il convient d'ailleurs de revenir sur les précisions de Cung Giu Nguyen au sujet de son activité littéraire :

Bref, et selon des interprétations psychanalytiques, ma création littéraire est due à la pulsion intérieure. Il se peut que je sois émotif, sensible, sentimental, lascif, voluptueux et quelque peu apathique, indolent, passible. Également introverti, je suis enclin à me replier sur moi-même, à réfléchir plutôt qu'à agir. Je suis inhibé et tyrannisé de sorte que j'accepte la douleur en la transformant en jouissance, dans la réalité ou dans l'imaginaire. Cela est d'ailleurs de la compensation, et avec la pression contraire, il apparaît donc une expulsion qui est d'autant plus forte qu'on est trop introverti et longtemps interné (cette violence peut ne pas se représenter par des gestes mais s'exprime dans plusieurs termes apparemment souples et agiles) [...]. Plus précisément, j'ai besoin de chasser mon "fantôme » de l'intérieur, d'exposer mon univers fantastique, de briser ma solitude, bien que ma voix ne retrouve pas sa réponse. (Nguyen Huu Thu, 1999, p. 27 ; je traduis)

L'écriture romanesque répond ainsi au défoulement. Cette affirmation de l'écrivain s'inscrit manifestement dans le cadre d'une écriture fantastique. Aussi, du point de vue psychanalytique, s'agit-il d'une expression de la fissilité du moi. Le Boujoum devient ainsi l'écriture de ce moi refoulé et clivé ainsi que le lieu où se manifeste une certaine dysphorie psychologique et morale, vécue par l'auteur comme une réalité. En effet, Cung Giu Nguyen fait entrer l'écriture du Boujoum dans un espace de l'absence et du vide où jaillissent l'amertume, la 
dépossession et le tourment du sujet. Le romancier est invité à vivre cette expérience comme une absurdité de l'existence afin de chercher une voie à l'intérieur de son acte d'écriture. Ainsi conçu, Le Boujoum n'est pas seulement un appel au secours sans réponse, comme dans Le Domaine maudit. Il n'est pas non plus le simple isolement d'un intellectuel marginal, comme dans Le Fils de la baleine. Il est la retranscription d'une solitude absolue, d'une solitude horizontale et verticale. Il s'agit d'une solitude et d'une désolation dans l'espace de la densité des figures humaines, dont la communication s'anéantit, laissant sortir un monde blessé et confus où se caractérise la tragédie de l'existence. De ce point de vue, Cung Giu Nguyen pratique une écriture de la pensée, selon laquelle le roman se prend pour un espace désert de signes, de mots et du langage conventionnel. De même, Le Boujoum évoque le langage d'un « effondrement » qui dépasse même l'expérience personnelle de la "déception », car cet effondrement entraîne une angoisse qui nous rappelle la désolation nietzschéenne et heideggérienne :

Le désert croît... Ce qui veut dire: la désolation s'étend. Désolation est plus que destruction. Désolation est plus sinistre qu'anéantissement. La destruction abolit seulement ce qui a crû et qui a été édifié jusqu'ici. Mais la désolation barre l'avenir à la croissance et empêche toute édification. (Heidegger, 1959, p. 36)

«L'effondrement » que l'auteur du Boujoum suscite ici est lié à la réalité de sa souffrance dans la quête désespérée d'une voie lisible ou d'une signification, de sorte qu'il devient inconscient chez le sujet comme forme de transcendance ou de dépossession :

L'enlisement semble m'épargner : l'amas de terre, un soufflé au chocolat, plutôt au moka, spongieux, a gardé un peu de consistance pour épargner aux masses spécifiques qui s'y 
égareraient le privilège de s'engloutir et de se perdre dans une masse plus grande. Vomi par la terre, sans raison, refusé, proclamé inapte au service de la légion infernale, classé ordure ou rebut, le corps cherche un axe. (Cung Giu Nguyen, 2002, p. 12)

Enfoncé dans un "gouffre » dont il fait l'expérience, le sujet cherche en vain à s'interroger sur son repère existentiel, étant donné qu'il ne trouve plus de rapports vertical et horizontal :

Où est la verticalité ? et sa cousine et ennemie ? elles se sont enfuies, emmenant ou emmenées, réconciliées, inverties, incestueuses, dans l'assortiment provisoirement inutile des cordonnées et des abscisses, des paraboles et des asymptotes, des angles et des perpendiculaires, des courbes, des fonctions sans fonctionnement. (ibid.)

Avec toute sa dimension philosophique de l'existence humaine, Le Boujoum conduit le lecteur à une écriture de l'allusion et de la métaphore qui condense le langage codé et symbolisé. Cette écriture énigmatique fait de Cung Giu Nguyen un romancier-révélateur, dont l'acte d'écriture ne consiste pas en une simple description de son époque, mais en un présage ou une initiation à la réflexion sur la condition existentielle ainsi que sur la démarche historique de l'homme sur terre.

Pour autant, ce roman résulte d'une écriture de la quête, où l'homme marche seul au milieu du désert des signes et des termes. C'est par cette écriture qu'on découvre « une réalité ambiguë », car, selon Barthes (1953, p. 16), cette écriture naît incontestablement d'une confrontation de l'écrivain et de sa société, et de cette finalité, elle renvoie l'écrivain, par une sorte de transfert tragique, aux sources instrumentales de sa création. Dans cette «initiative des mots» et incarné dans le personnage de Calame, un scribe dont la littérature est le seul 
espoir de l'existence, Cung Giu Nguyen témoigne d'une configuration de son langage énigmatique :

Car avec Calame, les mots qu'il employait n'avaient pas toujours le sens qu'on leur prête communément. L'obsession qui le hantait, celle de voir le monde comme une farce colossale, le prédisposait à la plaisanterie, au canular, au brouillage des cartes, à la destruction du langage familier au nom d'un autre langage qu'il pensait être plus efficient, plus communicatif, plus proche du réel. (Cung Giu Nguyen, 2002, p. 642)

Quel est ce «réel» dont l'auteur veut parler et qui demande «un autre langage » si ce n'est l'espace où l'homme est oublié et où le langage subit le « chaos des formes »? Aussi le romancier a-t-il la mission de "retrouver la fraîcheur d'un état neuf du langage » bien que ce processus finisse par creuser ses propres ornières, par créer ses propres lois, comme le constate Barthes (1953, p.55). C'est dans cet état neuf du langage que son expression poétique atteint une nouvelle dimension, un pouvoir réflexif et que son expressivité se révèle intemporelle. Ainsi, le chant d'Amdo et les soliloques de Pergorain, par la flûte, constituent-ils les quintessences de cette expressivité au point qu'ils affranchissent la réalité ordinaire pour se retranscrire dans une mémoire pérenne. Cette mémoire retrace les trajectoires du sujet apparemment ignoré au sein de la société sensible.

Le Boujoum interprète certes la précarité et l'incertitude du langage de la communication ordinaire ainsi que sa monotonie, son ambiguïté, mais derrière ces apparences sensibles se profile une écriture transparente ou « une écriture neutre » qui permet à l'écrivain d'entrer en relation avec une autre réalité profonde, de créer un monde fantastique. 


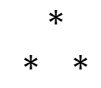

Du Fils de la baleine, par Le Domaine maudit et au Boujoum, Cung Giu Nguyen conserve la position d'un écrivain-philosophe qui réfléchit sans cesse à l'existence humaine et à ses conditions atroces. Ses romans se cristallisent par une démarche de transcendance et témoignent clairement d'une attitude de l'auteur devant la vie : la foi en l'art. Ils manifestent également une expérience et une expérimentation avec lesquelles la quête de signification finit par triompher dans l'impassibilité :

Je demeurerai à mon poste. Et s'il faut connaître une nouvelle expérience, eh bien, tant pis, rien ne me surprend plus, même le mariage du ciel et de l'enfer, même les noces de l'escargot et du requin, même la hantise du Boujoum, de ce qui se sent et ne s'explique pas, de cette obsession du sens tissé de non-sens. N'était-on pas confondu puis assuré devant quelque chose d'analogue? (Cung Giu Nguyen, 2002, p. 653)

Il est d'ailleurs intéressant de remarquer que, du premier roman au troisième, le lecteur aperçoit chez Cung Giu Nguyen une véritable évolution et une transformation remarquable de l'écriture qui, progressivement, correspond aux divers états où l'auteur vit son expérience du temps historique et de l'espace précaire. Mais l'espace intérieur s'étend dans son œuvre. L'espace immanent croît d'un roman à l'autre. Autrement dit, l'écriture romanesque de Cung Giu Nguyen est un reflet infini du monde intérieur et profond qui est plus vaste que le monde sensible de la société. Aussi l'acte d'écriture vise-t-il, pour Cung Giu Nguyen, à définir et à reconstruire sa position à travers la conceptualisation des personnages, comme le souligne Blanchot : 
Quand écrire, c'est se livrer à l'interminable [...]. L'idée de personnage [...] n'est qu'un des compromis par lesquels l'écrivain, entraîné hors de soi par la littérature en quête de son essence, essaie de sauver ses rapports avec le monde et avec lui-même. (1955, p. 21)

"Se livrer à l'interminable » pour être entraîné hors de soi devient donc chez Cung Giu Nguyen une forme d'alchimie, une descente dans un espace profond et vertical, de sorte que cette recherche se transforme en une projection de l'inconscient lorsque l'auteur crée vers la fin du roman son personnage de Kate, attaché à Calame comme un symbole et une empreinte du temps, comme « une mémoire, un regard, un désir, une lettre d'amour ».

\section{Bibliographie}

Assoun, Paul-Laurent. (1996), Littérature et psychologie, Paris, Ellipse.

BARTHES, Roland. (1953), Le Degré zéro de l'écriture, Paris, Seuil. BlAnCHOT, Maurice. (1955), L'Espace littéraire, Paris, Gallimard.

Cung, Giu Nguyen. (2002), Le Boujoum, Dallas-Texas, Cunggiunguyen Center Publications.

—. (1994), Thái huyền, Californie, Dai Nam. 
—. (1978 [1956]), Le Fils de la baleine, Québec, Naaman.

-. (1961), Le Domaine maudit, Paris, Fayard.

HEIDEGGER, Martin. (1959), Qu'appelle-t-on penser ?, Paris, PUF.

NGUYEN, Huu Thu. (1999), « Giáo sư Cung Giũ Nguyên, một nhà trí thức Huế đáng kính » (Professeur Cung Giu Nguyen, un intellectuel vénérable de Hué), Tuyển tập nhớ Huế, $\mathrm{n}^{\circ}$ 10, p. 21-31.

ROBERT, Marthe. (1972), Roman des origines et origines du roman, Paris, Grasset.

\section{Résumé}

Si la littérature résulte d'une réalisation esthétique et poétique de l'écriture, elle est aussi sensible à des événements sociaux et à des conditions humaines. Les œuvres d'auteurs vietnamiens francophones s'inscrivent dans cette perspective en s'affrontant souvent au monde bouleversé et dramatique ou à un espace scénographique chaotique où l'individu évolue. Cung Giu Nguyen, une des grandes figures de la littérature francophone vietnamienne, témoigne clairement de sa foi dans l'art de sorte que son écriture manifeste la volonté de métamorphoser la réalité vécue. Cet article se propose d'examiner la mise en scène de l'écriture comme une projection spatiotemporelle en rapport avec le sujet (fictif ou de l'écriture), ce qui, du point de vue psychanalytique, permettrait d'éclairer le moi clivé de l'écrivain. 


\begin{abstract}
If literature is the result of an aesthetic and poetic realization of writing, it is also sensitive to social events and human conditions. Works of Francophone Vietnamese authors are firmly rooted in this perspective often confronting the reversed and dramatic world or chaotic scenographic space where the individual evolves. Form of fiction writing, Giu Cung Nguyen, one particular figure of French literature, evidence, most visibly, his faith in art so that his writing shows a power to transform the reality. This article aims to clear this idea by examining the staging of writing fiction as a spatiotemporal projection in the psychoanalytic perspective. This leads us to analyze the ego's cleavage of writer.
\end{abstract}

